\title{
Response force is sensitive to the temporal uncertainty of response stimuli
}

\author{
STEFAN MATTES and ROLF ULRICH \\ University of Wuppertal, Wuppertal, Germany
}

\begin{abstract}
Three experiments examined whether temporal uncertainty about the delivery of a response stimulus affects response force in a simple reaction time (RT) situation. All experiments manipulated the foreperiod; that is, the interval between a warning signal and the response stimulus. In the constant condition, foreperiod length was kept constant over a block of trials but changed from block to block. In the variable condition, foreperiod length varied randomly from trial to trial. A visual warning and response stimulus were used in Experiment 1; response force decreased with foreperiod length in the variable condition, but increased in the constant condition. This result is consistent with the hypothesis that responses are less forceful when the temporal occurrence of the response stimulus is predictable. In a second experiment with an auditory warning signal and a response stimulus, response force was less sensitive to foreperiod manipulations. The third experiment manipulated both the modality and the intensity of the response signal and employed a tactile warning signal. This experiment indicated that neither the modality nor the intensity of the response signal affects the relation between response force and foreperiod length. An extension of Näätänen's (1971) motor-readiness model accounts for the main results.
\end{abstract}

In a typical reaction time (RT) experiment, a warning signal precedes the imperative response stimulus. ${ }^{l}$ Since the pioneering study of Woodrow (1914), it has been repeatedly documented that RT is strongly affected by the interval between the warning signal and the stimulus, that is, the foreperiod. According to Niemi and Näätänen (1981), the warning signal is used as a temporal reference for response preparation. Subjects estimate the point in time when the stimulus is delivered and try to synchronize response preparation with stimulus occurrence. Predictability of the stimulus and, hence, optimal response preparation depend on several foreperiod factors (for a review, see Niemi \& Näätänen, 1981). A consistent finding is that factors favoring the exact prediction of the stimulus yield especially short RTs (e.g., constant foreperiods compared with variable ones). Some RT theorists (e.g., Sanders, 1990) assume that foreperiod manipulations affect the duration of motor processing, that is, the motoric portion of RT.

Given that foreperiod factors operate at a motoric level, one may ask whether these factors influence not only RT but also the exerted force in making the response. Recent studies (Giray, 1990; Jaśkowski \& Verleger, 1993) have tackled this question. Giray employed variable foreperiods in a simple RT task. In each trial, a foreperiod length was

This work was supported by the Deutsche Forschungsgemeinschaft (UL 88/103). We appreciate the helpful comments of Hartmut Leuthold, Jeff Miller, and two anonymous reviewers. We are also indebted to Hiltraut Müller-Gethmann for assistance in data collection and helpful comments on an earlier draft of this manuscript. Correspondence should be addressed to R. Ulrich, General Psychology I, Fachbereich 3, University of Wuppertal, Gauss-Strasse 20, D-42097 Wuppertal, Germany (e-mail: ulrich@uni-wuppertal.de). randomly sampled from the exponential distribution. This foreperiod distribution is called "non-aging," because the momentary tendency for the stimulus to occur is not affected by the time elapsed since the appearance of the warning signal. Giray recorded the maximum attained response force in a single trial. This measure decreased with increasing foreperiod duration. Responses were forceful and slow at short foreperiods and less forceful and fast at long foreperiods. He attributed this foreperiod duration effect mainly to an increase of subjective expectancy (i.e., subjective probability of the immediate delivery of the stimulus) as the foreperiod is prolonged. Jaśkowski and Verleger reached a similar conclusion in a nonstandard RT foreperiod paradigm in which a clock provided temporal information to the subject about stimulus delivery.

Both studies suggest that a response is less forceful when a subject expects the stimulus. For three reasons, however, it would be premature to generalize this conclusion to typical experimental situations. First, a standard RT paradigm provides no external temporal information about stimulus delivery. Therefore, it is difficult to generalize Jaśkowski and Verleger's (1993) conclusion to a standard foreperiod design. Second, non-aging foreperiod distributions are seldom employed in RT studies. Therefore, it is problematic to extend Giray's (1990) conclusion to the majority of RT studies. Third, it is somewhat puzzling that these two studies reported similar results, even though different foreperiod distributions (aging vs. non-aging) were employed. It is a well-established fact in RT research that the nature of the foreperiod distribution exerts a subtle influence on stimulus predictability and thus on the foreperiod duration effect (Niemi \& Näätänen, 1981; Requin, Brener, \& Ring, 1991). Because both studies reported a 
decrease of peak force with foreperiod length, it is unclear whether stimulus predictability systematically influences the relation between foreperiod and peak force, as has often been documented for the relation between foreperiod and RT. Hence, if the predictability of the stimulus modulates the relation between foreperiod and force-as it does the relation between foreperiod and RT - this would strengthen the hypothesis that response force depends on subjective expectancy, or, more specifically, on the degree of response preparation. ${ }^{2}$ The present paper addresses this issue within the framework of standard foreperiod paradigms. This work may not only contribute to a better understanding of the mechanisms underlying force production, but also improve the interpretation of response force as an inferential tool in cognitive psychology (Abrams \& Balota, 1991; Coles, Gratton, Bashore, Eriksen, \& Donchin, 1985; Giray \& Ulrich, 1993; Kantowitz, 1973; Miller, Franz, \& Ulrich, 1997; Mordkoff, Miller, \& Roch, 1996; Ulrich \& Mattes, 1996).

The first two experiments engage two conditions of foreperiod regularity to manipulate the predictability of stimulus occurrence. The same set of foreperiod durations $(0.50,1.75$, and $3.00 \mathrm{sec})$ was used in both conditions. In the constant condition, one foreperiod duration was used throughout a block of trials but changed from block to block. In the variable condition, a foreperiod length was randomly sampled in each trial from the aforementioned set of foreperiod durations. A common finding is that RT progressively increases with foreperiod duration in the constant condition but decreases in the variable condition. This modulation of the relation between foreperiod and RT reflects different strategies of response preparation (see Niemi \& Näätänen, 1981; Requin et al., 1991). The RT increase in the constant condition presumably reflects a progressive deterioration of the subject's ability to estimate stimulus occurrence when the foreperiod is lengthened, which in turn impairs the synchronization of response preparation with the onset of the stimulus at longer foreperiods. In the variable condition, time estimation is not a useful strategy for synchronizing response preparation with stimulus onset. This condition constitutes an aging foreperiod distribution because the objective probability of stimulus delivery increases with the time elapsed since the warning signal. The subject somehow learns to use this probability increase and accomplishes a growth of response preparation associated with this increase. In sum, RT studies provide ample evidence that response preparation diminishes with foreperiod length in the constant condition and increases with foreperiod length in the variable condition. Hence, Giray's (1990) and Jaśkowski and Verleger's (1993) expectancy hypothesis implies that peak force should increase with foreperiod length in the constant condition and decrease in the variable condition.

In Experiment 1, visual stimulations were used and the results were as predicted. Experiment 2 employed auditory stimulations to assess whether arousal effects would reduce the foreperiod effect obtained in Experiment 1. This was found to be the case. Experiment 3 was designed to determine whether the sensory modality or the intensity of the stimulus modulates the foreperiod effect on both RT and response force. It was found that neither modality nor intensity modulated this effect.

\section{EXPERIMENT 1}

This experiment employed a factorial combination of three foreperiod durations $(0.50,1.75$, and $3.00 \mathrm{sec})$ and two levels of foreperiod regularity (constant vs. variable foreperiod). This design systematically manipulates the predictability of the response stimulus. As explained in the introduction, high predictability is favored by short and constant foreperiods, whereas low predictability is favored by short and variable foreperiods. Both RT and peak force were expected to decrease with stimulus predictability.

\section{Method}

Subjects. Five female and 13 male subjects (mean age $=25.3$ years) participated in a single session. They were paid for their cooperation and were naive about the experimental hypothesis. All subjects claimed to be right-handed.

Apparatus and Stimuli. Subjects were seated in a dimly illuminated room. A microcomputer controlled signal presentation and recorded response force. There was a background noise of $36 \mathrm{~dB}(\mathrm{~A})$ at the level of the subject's ear. The warning signal consisted of a yellow cross appearing in the center of the computer screen for $300 \mathrm{msec}$. The response stimulus was a white patch $(9.5 \times 11.2 \mathrm{~cm})$. The duration of this stimulus was $150 \mathrm{msec}$, and its intensity was $3.1 \mathrm{~cd} / \mathrm{m}^{2}$.

Subjects responded with a brief flexion of the right index finger. The force of this flexion was measured by force-sensitive keys similar to telegraph keys. A leaf spring $(110 \times 19 \times 2 \mathrm{~mm})$ was held by an adjustable clamp at one end, while the other end remained free. Any force applied to the leaf spring at the free end was reflected by an analogous electrical signal that was digitized with a sampling rate of $500 \mathrm{~Hz}$. A force of $10 \mathrm{~N}$ bent the free end by about $1 \mathrm{~mm}$. The resolution of this device was about $0.02 \mathrm{~N}$. The subject's forearm rested comfortably on a board that provided full forearm support.

Procedure. A trial started with the presentation of the warning signal. After a given foreperiod duration, the response stimulus was administered. Sampling of response force began $150 \mathrm{msec}$ before stimulus onset and continued for $2,000 \mathrm{msec}$. This sampling duration was long enough to cover the whole force-time function of the response. The interval between stimulus onset and the point at which the response force reached the criterion of $50 \mathrm{cN}$ was considered as RT. There was no constraint on any characteristic of the response other than to exceed the required criterion level as fast as possible after stimulus onset while avoiding anticipations. Subjects were informed about response errors at the end of the sampling duration. If the RT was shorter than $100 \mathrm{msec}$ (false alarm) or longer than $1,000 \mathrm{msec}$ (miss), corresponding information was provided on the computer screen. Those trials were considered as response errors and were discarded from the data analysis.

A session lasted about $45 \mathrm{~min}$ and consisted of 12 blocks of 36 trials each. In the variable condition, foreperiod duration varied randomly from trial to trial (i.e., $0.5,1.75$, or $3.0 \mathrm{sec}$ ). In the constant condition, each of the three foreperiod durations was run in a separate block. Order of conditions was balanced over subjects according to a Latin square. There were always two identical blocks in succession, the first of which was considered practice and was excluded from the data analysis. After each block, feedback about mean RT and response errors appeared on the computer screen. Subjects were asked to provide responses quickly but to avoid response errors. 


\section{Results}

A separate two-way analysis of variance (ANOVA) with the factors of foreperiod duration and foreperiod regularity (constant vs. variable foreperiods) was performed for RT, peak force, and on the arcsin transformed (Winer, 1971) proportions of false alarms.

Response errors. The percentages of false alarms (RT $<100 \mathrm{msec}$ ) and misses (RT $>1,000 \mathrm{msec}$ ) were 3.7 and $1.2 \%$, respectively. Significantly more false alarms resulted for constant than for variable foreperiods $[F(1,17)=$ $50.4, p<.001]$. False alarms decreased with foreperiod duration in the constant condition but were almost uniform in the variable condition $[F(2,34)=36.7, p<.001]$; the respective figures were $15.7 \%, 3.9 \%$, and $1.7 \%$ in the constant condition and $0.2 \%, 0.2 \%$, and $0.3 \%$ in the variable condition. This error pattern is consistent with the notion that subjects were highly prepared in the constant foreperiod condition and therefore especially prone to anticipation errors.

Reaction time. Figure 1 depicts mean RT (upper panel) and peak force (lower panel) as a function of foreperiod duration and foreperiod regularity with corresponding $S E s{ }^{3}$ Foreperiod duration revealed no significant main effect $[F(2,34)=1.6, p=.220]$. As expected, constant foreperiods yielded significantly faster RTs $(M=191 \mathrm{msec})$ than did variable ones $[M=223 \mathrm{msec} ; F(1,17)=58.7$, $p<.001]$. However, a significant interaction of foreperiod duration $\times$ regularity indicated that this RT difference diminished with foreperiod length $[F(2,34)=38.6$, $p<.001$ ], although it was significant at each level of foreperiod length $(t$ test, all $p s<.026)$. Separate one-way ANOVAs with the factor of foreperiod duration were computed for each condition of foreperiod regularity. Both ANOVAs yielded highly significant effects of foreperiod length on RT. As expected, mean RT increased with foreperiod duration in the constant condition but decreased in the variable condition.

Response force. In each trial, peak force was determined on the basis of the recorded force-time function in order to assess potential effects on response force. Other force measures could also be used (e.g., the area under the force impulse), but these tend to be highly correlated with and slightly noisier than peak force (cf. Giray \& Ulrich, 1993).

The ANOVA results for peak force resembled those for RT. There was no main effect of foreperiod duration on peak force $(F<1)$. More forceful responses were observed for variable than for constant foreperiods, although this effect just failed to reach statistical significance $[F(1,17)=$ $3.8, p=.067]$. The significant interaction of foreperiod duration $\times$ foreperiod regularity showed that this difference shrank as foreperiod duration increased $[F(2,34)=$ $38.6, p<.001]$. Additional $t$ tests revealed a significant difference $(p=.022)$ for the shortest but not for the two longer foreperiod durations.

Separate one-way ANOVAs with the factor of foreperiod length were also computed for each foreperiod condition. Factor foreperiod length produced a highly significant effect for variable foreperiods $[F(2,34)=14.2$,
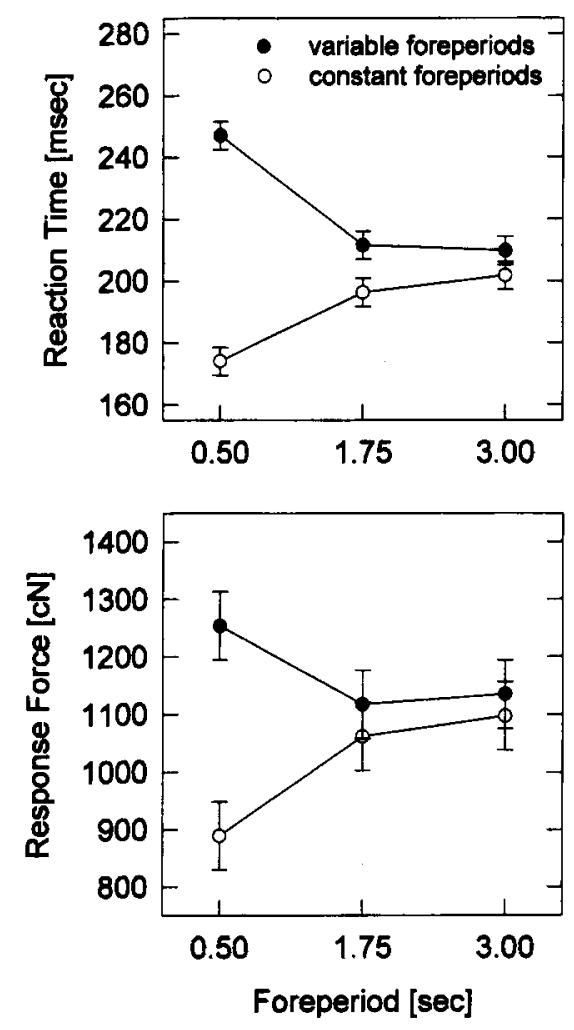

Figure 1. Experiment 1: reaction time (upper panel) and peak force (lower panel) as a function of foreperiod regularity and length. The error bars indicate the $S E s$, which were estimated from the pooled error terms of the respective ANOVAs.

$p<.001]$ and approached statistical significance for constant foreperiods $[F(2,34)=3.2, p=.052]$.

\section{Discussion}

This experiment successfully replicated the finding that the temporal predictability of the response signal enhances response speed. As suggested by the notion of stimulus predictability, RT increased with foreperiod length in the constant condition but decreased with foreperiod length in the variable condition (Niemi \& Näätänen, 1981). Furthermore, longer RTs resulted for variable than for constant foreperiods. This difference has been observed in other studies (e.g., Hohle, 1965; Manning \& Hammond, 1990) and may be attributed to the generally greater temporal uncertainty level associated with variable foreperiods.

A similar pattern of results was observed for response force. Peak force decreased as the temporal predictability of the stimulus increased. In sum, the obtained results strongly support the idea that response force decreases with stimulus predictability. This result agrees with the claim made by RT theorists (e.g., Sanders, 1990) that foreperiod factors operate at a motoric level in the information processing system. Presumably, stimulus predictability permits an efficient adjustment of motor readiness, implying not only fast but also economical responses; this will be discussed in more detail in the General Discussion. 


\section{EXPERIMENT 2}

Prolongation of foreperiod duration has a smaller effect on RT when the warning signal (Posner, Nissen, \& Klein, 1976) or the response stimulus (Sanders \& Wertheim, 1973) is auditory than when both are visual. Loud auditory stimulation is generally assumed to exert an immediate arousal effect that supplies the response conduction process with extra energy and thus facilitates the execution of a response. Some RT theorists (e.g., Molenaar \& van der Molen, 1986; Sanders, 1983) have postulated that immediate arousal effects bypass central computational stages and influence more or less directly the motor system. The latter view in particular suggests that the foreperiod effect on response force should be attenuated or even eliminated in a situation in which auditory stimulation is employed. Therefore, in Experiment 2, we sought to examine whether response force, like RT, is less sensitive to foreperiod manipulations in an auditory RT task in which both the warning signal and the stimulus are tones.

\section{Method}

Subjects. A fresh sample of 18 female and 18 male subjects (mean age $=26.6$ years) participated in a single session. They were paid for their cooperation and were naive about the experimental hypothesis. All subjects but 1 claimed to be right-handed.

Apparatus and Stimuli. The apparatus and stimuli were the same as those in Experiment 1 except for sense modality. Both the warning signal and the stimulus were presented binaurally over headphones. The duration of the warning signal was $300 \mathrm{msec}$ and its sound level was $60 \mathrm{~dB}(\mathrm{~A})$. The sound level of the stimulus was $80 \mathrm{~dB}(\mathrm{~A})$ and its duration was $150 \mathrm{msec}$.

Procedure. The procedure was identical to that used in Experiment 1 .

\section{Results}

Response errors. The percentages of false alarms (RT $<100 \mathrm{msec}$ ) and misses (RT $>1,000 \mathrm{msec}$ ) were $5.0 \%$ and $0.6 \%$, respectively. The obtained pattern of false alarms agreed with the one in Experiment 1 . Subjects again produced more false alarms in the constant condition than in the variable condition $[F(1,35)=39.8, p<$ $.001]$. Furthermore, false alarms again decreased with foreperiod duration in the constant condition, but were again almost uniform in the variable condition $[F(2,70)=$ $30.9, p<.001]$; the corresponding percentages were $15.6 \%, 4.8 \%$, and $3.7 \%$ in the constant condition and $2.2 \%, 1.7 \%$, and $2.2 \%$ in the variable condition.

Reaction time. Figure 2 shows RT (upper panel) and peak force (lower panel) as a function of foreperiod length and foreperiod regularity. The overall mean RT was shorter than that in Experiment 1 (185 vs. $207 \mathrm{msec}$ ). As in Experiment 1, constant foreperiods yielded significantly faster RTs than did variable ones $[F(1,35)=$ $34.4, p<.001]$. However, the mean RT difference was $24 \mathrm{msec}$, which is smaller than that found in Experiment 1. In contrast to the previous experiment, however, this difference decreased only slightly with foreperiod duration $[F(2,70)=3.7, p=.03]$. In sum, then, changing the sen-
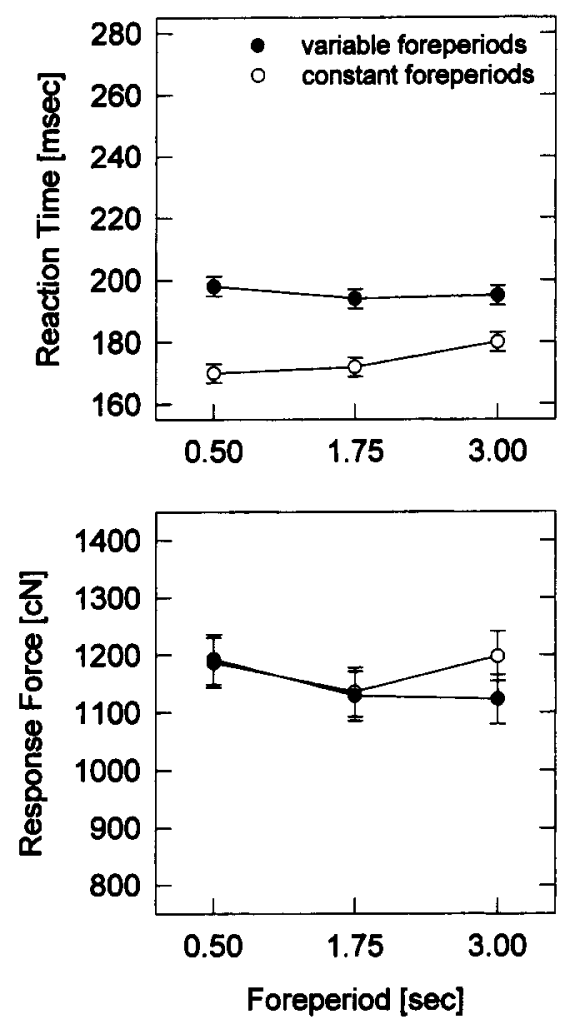

Figure 2. Experiment 2: reaction time (upper panel) and peak force (lower panel) as a function of foreperiod regularity and length. The error bars indicate the $S E s$, which were estimated from the pooled error terms of the respective ANOVAs.

sory modality of the warning signal and the response stimulus from the visual to the auditory mode greatly reduced the influence of the foreperiod manipulation on RT.

Response force. A two-way ANOVA with the factors of foreperiod length and foreperiod regularity yielded no significant effect on peak force.

\section{Discussion}

The present RT results confirm the finding that auditory stimulation attenuates the effect of foreperiod on response speed. These results contrast with those of Experiment 1 , in which foreperiod length strongly affected RT. These different patterns are consistent with the claim that auditory stimulation produces automatic alerting/ arousal (Nissen, 1977; Sanders, 1975, 1983), eliminating foreperiod effects on RT. Interestingly, however, an almost identical pattern of results was obtained for response force. Thus, this experiment indicates that an auditory RT task not only attenuates the effects of foreperiod on RT, but may also eliminate those effects on response force.

\section{EXPERIMENT 3}

The results of the preceding experiments suggest that the temporal uncertainty about stimulus occurrence has a stronger effect on RT and response force for visual 
than for auditory stimuli. This differential effect might be due to the arousing property of auditory stimuli. However, this conclusion seems premature for two reasons. First, the loud stimulus in Experiment 2 is likely to have been subjectively much more intense than the visual one in Experiment 1. It is therefore possible that the loud tone might have elicited a "reflex" response that appeared to be faster and more forceful. Second, the two experiments also differ with respect to the modality of the warnings. Experiment 1 employed a visual warning signal, whereas Experiment 2 employed an auditory warning signal. It is known that an auditory warning signal exerts an elevated state of phasic alertness (Bertelson \& Tisseyre, 1969; Ulrich \& Mattes, 1996), and this might have reduced the foreperiod effect on RT and response force.

In Experiment 3, we sought to eliminate these potential shortcomings. First, this experiment employed two stimulus intensity levels in each sense modality with approximately psychophysically matched intensity levels (see Stevens, 1955). The use of two levels enabled us to separate the effects of stimulus modality from those of stimulus intensity. Second, a tactile stimulation served as a neutral warning signal in both modality conditions. To keep the session duration constant across experiments, only the variable foreperiod condition was included. One group of subjects was assigned to the auditory condition, and a second group was assigned to the visual condition. Therefore, this experiment provided a factorial combination of foreperiod length $(0.50,1.75$, and $3.00 \mathrm{sec}$ ), stimulus intensity (low vs. high), and stimulus modality (visual vs. auditory stimulus), allowing investigation of the following questions: (1) Does the foreperiod effect on peak force diminish with increasing stimulus intensity? and (2) Does the foreperiod effect depend on stimulus modality when a neutral warning signal is employed?

\section{Method}

Subjects. A fresh sample of 27 female and 21 male subjects (mean age $=24.8$ years) participated in a single session. They were paid for their cooperation and were naive about the experimental hypothesis. All but 2 subjects claimed to be right-handed. In order to keep the present design maximally comparable to Experiments 1 and 2 , half the subjects received the visual stimulus and half received the auditory stimulus. Subjects were randomly assigned to each group.

Apparatus and Stimuli. The apparatus and stimuli were the same as those in Experiments 1 and 2 except for the warning signal and the intensity levels of the stimuli. A soft yet clearly noticeable touch on the left index finger served as the warning signal. The subject's index fingertip rested in a hollow. In its middle there was a hole in which a movable pestile (diameter $2 \mathrm{~mm}$ ) was at rest position. A magnet moved this pestile upward within a few milliseconds and removed it after $50 \mathrm{msec}$. In the working position, the pestile projected beyond the surface of the hollow by approximately $2 \mathrm{~mm}$. This mechanical stimulation was not audible.

Except for stimulus intensity, the visual and the auditory stimuli were identical to those used in Experiments 1 and 2, respectively. The visual intensity levels were 0.3 and $100 \mathrm{~cd} / \mathrm{m}^{2}$, and the auditory intensity levels were 55 and $85 \mathrm{~dB}(\mathrm{~A}) .^{4}$

Procedure. This was identical to that of the previous experiments except that stimulus intensity and foreperiod duration varied randomly from trial to trial throughout the whole session.

\section{Results}

Response errors. The percentages of false alarms (RT $<100 \mathrm{msec}$ ) and misses ( $\mathrm{RT}>1,000 \mathrm{msec}$ ) were very low $(0.4 \%$ and $0.9 \%$, respectively). In the visual condition, the percentage of false alarms was 0.9 ; in the auditory condition, this percentage was $1.0 \%$. These figures were too low to permit a meaningful statistical analysis.

Figure 3 depicts RT (upper panels) and peak force (lower panels) as a function of foreperiod length, stimulus intensity, and stimulus modality. The left panels show the results for the visual condition, and the right panels show the results for the auditory condition. Separate threeway ANOVAs with the factors of foreperiod length, stimulus intensity, and stimulus modality were performed for RT and peak force.

Reaction time. Responses were slightly faster in the auditory condition $(M=229 \mathrm{msec})$ than in the visual condition $(M=238 \mathrm{msec})$, although this difference was not significant $(F<1)$. As expected, shorter RTs resulted with strong stimuli $(M=220 \mathrm{msec})$ than with weak stimuli $[M=247 \mathrm{msec} ; F(1,46)=424.0, p<.001]$. This intensity effect was slightly larger for auditory than for visual stimuli $[F(1,46)=38.6, p<.001]$.

The ANOVA revealed a highly significant effect of foreperiod length on RT $[F(2,92)=45.6, p<.001]$. As expected, RT decreased with foreperiod length. Post hoc comparisons (Newman-Keuls) indicated that all means were significantly different from one another (the means were 244,231 , and $226 \mathrm{msec}$ for the foreperiod durations of $0.5,1.75$, and $3.0 \mathrm{sec}$, respectively). In addition, the significant interaction of modality and foreperiod length indicated that the foreperiod effect was larger with visual than with auditory stimuli $[F(2,92)=8.0, p=.001]$. There were no further significant effects.

Response force. Subjects in the auditory condition produced more forceful responses than did those in the visual condition. However, this difference was nonsignifcant $[F(1,46)=1.3, p=.269] . .^{5}$ Factor stimulus intensity revealed a highly reliable effect on peak force $[F(1,46)=$ $34.2, p<.001]$. Consistent with previous results (Angel, 1973), peak force was larger for intense $(M=1,006 \mathrm{cN})$ than for weak $(M=1,055 \mathrm{cN})$ stimuli. Moreover, this intensity effect was larger in the auditory than in the visual condition $[F(1,46)=9.0, p=.004]$.

As expected, peak force decreased as foreperiod length increased $[F(2,92)=68.6, p<.001]$. Post hoc comparisons (Newman-Keuls) showed that peak force was significantly larger for the shortest foreperiod than for the middle and longest foreperiods, which did not differ from each other ( $M=1,092,997$, and $1,002 \mathrm{cN}$, respectively). Contrary to our expectations, however, this foreperiod effect was significantly modulated neither by stimulus intensity $[F(2,92)=1.9, p=.160]$ nor by stimulus modality $[F(2,92)=1.2, p<.303]$. No other sources of variance approached significance.

We also carried out separate ANOVAs for each modality condition to determine whether the aforementioned significant main effects were also present in each condition. In both modality conditions, the main effects of fore- 

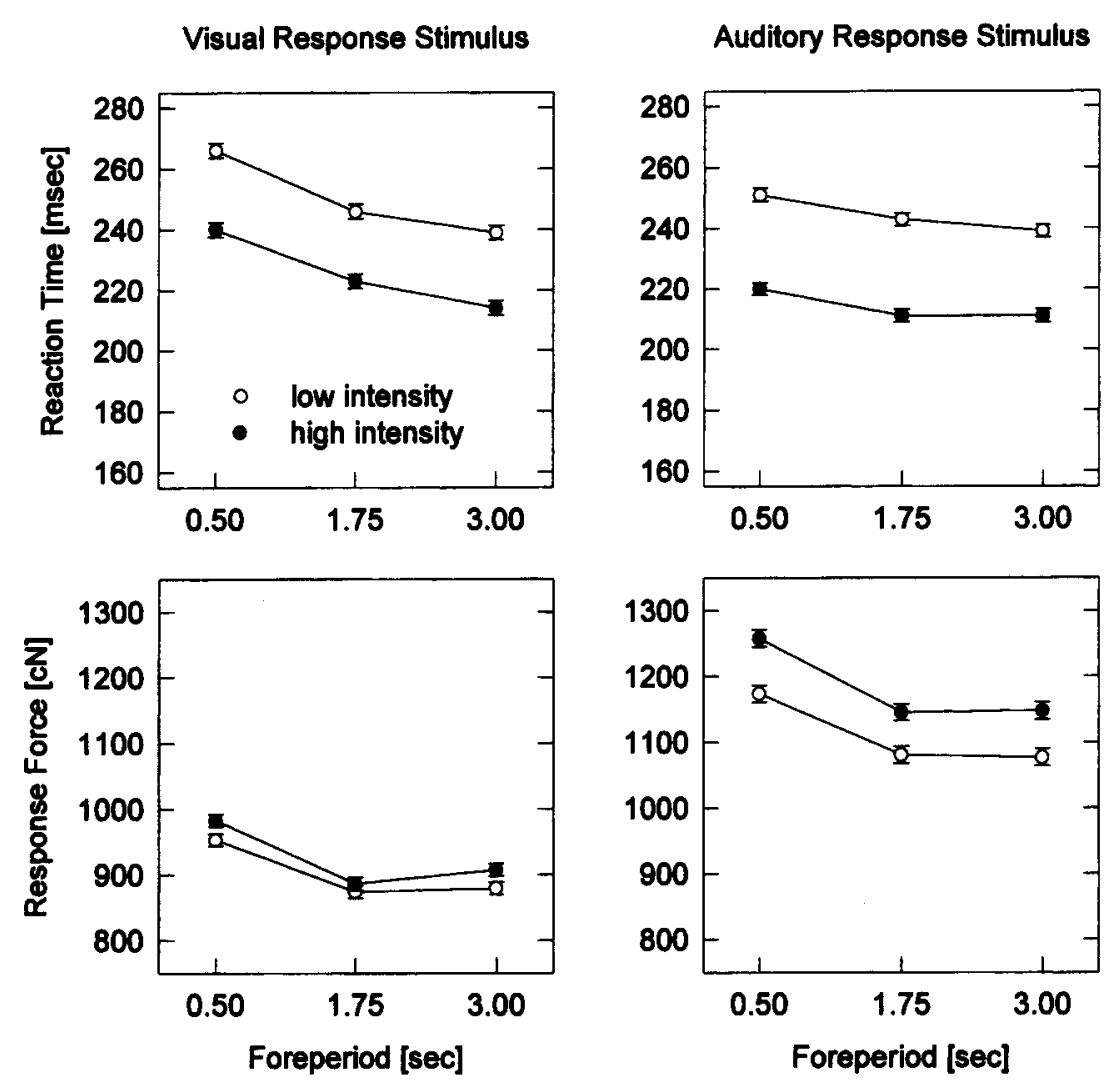

Figure 3. Experiment 3: reaction time (upper panels) and peak force (lower panels) as functions of foreperiod length, stimulus intensity, and stimulus modality (left panels = visual stimuli, right panels = auditory stimuli). The error bars indicate the $S E s$, which were estimated from the pooled error terms of a two-way ANOVA (response signal intensity $\times$ foreperiod length) for each panel. Therefore, the standard errors are appropriate for judging differences within each panel.

period length and stimulus intensity were highly significant (all $p \mathrm{~s}<.001)$.

\section{Discussion}

This experiment successfully replicated the finding that response speed increases with both the intensity and the predictability of the stimulus. Consistent with previous research (Sanders \& Wertheim, 1973), this effect was smaller for auditory than for visual stimuli, despite the fact that the overall mean RT was approximately the same in both modalities. Most importantly, however, a clear foreperiod effect on response force was obtained. This effect was influenced by neither the intensity nor the modality of the stimulus. In agreement with the expectancy hypothesis, peak force decreased with increasing foreperiod duration.

The present experiment provides strong evidence for the conjecture that the auditory warning signal in Experiment 2 strongly reduced the foreperiod effect on RT and eliminated it for peak force. This elimination might be attributed to the arousing property of the auditory warning signal, which might have affected the subject's state of re- sponse preparation. This interpretation is supported by a study of Ulrich and Mattes (1996). Their results showed that responses became more forceful and faster as the intensity of an auditory warning signal was increased.

\section{GENERAL DISCUSSION}

Previous studies have shown that responses in a simple RT task become more forceful when the foreperiod duration interval is shortened (Giray, 1990; Jaśkowski \& Verleger, 1993). The present results not only replicate this finding, but also expand it considerably. Experiment 1 employed a purely visual task in which both the warning and the response stimulus were visual. The results of this experiment showed that the foreperiod effect on response force and RT is not merely a function of foreperiod length, but is associated with the temporal predictability of the stimulus. As expected, response force increased with constant foreperiods but decreased with variable foreperiods. High predictability of temporal stimulus occurrence presumably allows for an efficient adjustment of motor readiness, leading to a fast and fru- 
gal response execution. Experiment 2 employed a purely auditory task in which both the warning and the response stimulus were auditory. In this task, the foreperiod effect on response force and RT was greatly reduced. Experiment 3 employed a bimodal task with a tactile warning signal and a response stimulus from another modality (visual or auditory). A clear foreperiod effect on response force and RT was found. Interestingly enough, the foreperiod effect on response force depended on neither the intensity nor the modality of the stimulus.

The present results clearly support the notion that foreperiod manipulations affect the force output of speeded responses. As noted in the introduction, this foreperiod effect seems to reflect an intrinsic motor adjustment and therefore substantiates the conclusion of RT theorists (Sanders, 1990) that the foreperiod effect operates at distal processing stages. However, this effect is subject to the nature of the warning signal. The auditory warning signal-but neither the visual nor the tactile warning signal--attenuated the foreperiod effect. Whether this finding can be attributed to the sensory modality of the warning signal per se, or merely to its perceived intensity level, is unclear, although such a modality difference seems to exist for RT (see, e.g., Posner et al., 1976). A psychophysical match of the perceived intensity of the auditory, visual, and tactile warning signals would be required to distinguish between these possibilities. Nevertheless, neither the modality nor the intensity of the response stimulus seems to affect foreperiod manipulations on response force, although such influences have sometimes been reported for RT (Niemi \& Lethonen, 1982; Sanders \& Wertheim, 1973; but see Bernstein, Chu, \& Briggs, 1973; Niemi, 1979; Sanders, 1977).

A model of response preparation originally proposed by Näätänen (1971) provides a tentative yet useful framework for integrating the major findings of this study. The variable of "motor readiness" constitutes the basic concept of this model. Motor readiness is conceived as the difference between excitatory motor commands and inhibitory motor commands. The subject establishes a criterion (i.e., the motor action limit), and when motor readiness reaches this limit, an overt response is inevitably triggered. Motor readiness fluctuates due to ongoing central correcting commands that maintain the desired level of motor preparation. Response speed depends on the momentary distance between motor readiness and the motor limit at which the stimulus is delivered. If this distance is small, fast responses will result. If subjects expect the stimulus, they will adjust motor readiness when they are close to the motor limit. The temporal accuracy of this adjustment depends on the predictability of stimulus occurrence. Thus, response speed will increase with stimulus predictability.

However, this hypothesis also suggests that response force decreases with predictability. When the subject is well prepared-that is, when motor readiness is close to the motor action limit at the very moment of stimulus de- livery - a small excitation increment is sufficient to trigger the response. However, when the subject is in a poor state of preparation, a larger excitation increment is needed to cross the motor limit. Because a fast response is required, the brain will employ more excitation than necessary to be on the safe side. It is plausible that the amount of excitation overshoot contributes to the force output of a response. For example, more motoneurons (Ulrich \& Stapf, 1984) or force units (Ulrich \& Wing, 1991) might be recruited than are necessary for launching an economical response.

This model may also account for the finding that greater false alarm rates were linked with fast RTs: When motor readiness is close to the motor limit, a small random increase of motor readiness can trigger a premature response. The response error pattern of Experiments 1 and 2 under the constant foreperiod condition clearly agrees with this prediction. This condition produced particularly fast RTs but also an increased number of premature responses, suggesting that motor readiness was especially close to the motor limit, thus provoking premature responses. In addition, and consistent with the model, the rate of premature responses decreased with foreperiod length in the constant condition. In contrast to the model's prediction, this error rate did not increase with foreperiod length in the variable condition. However, it seems plausible to assume that motor readiness in the variable condition was well below the action limit and therefore could not have influenced the false alarm rate. The few false alarms observed in this condition may be attributed to spurious observations rather than to errors emerging from response preparation (see Ulich \& Miller, 1994). In conclusion, the motor-readiness model holds that both RT and response force should decrease and false alarm rate should increase with stimulus predictability.

This framework does not directly account for the finding that auditory warning signals eliminated the foreperiod effect on response force and greatly reduced this effect on RT. However, it seems possible that auditory warning signals affect the response conduction process via a separate pathway that bypasses the regular information processing route from a stimulus to its associated response (Miller et al., 1997; Sanders, 1983; Ulrich \& Mattes, 1996). This separate route directly connects the sensory input with the motor output. Activation of this route does not elicit a response, but rather supplies the response conduction process with extra energy. It seems reasonable that this extra yet uncontrollable input into the response conduction process may seriously interfere with an effort to adjust motor readiness. More research is needed to assess this specific point.

Response force is usually not analyzed in the field of information processing (Meyer, Osman, Irwin, \& Yantis, 1988 ) and is therefore less well understood than RT (Luce, 1986). The motor-readiness model may provide a useful first step toward a better understanding of the mechanisms underlying response force. Although we are still 
far from a complete understanding of these mechanisms, the present and other studies have isolated two important factors determining the force level of a response: temporal uncertainty of the response stimulus and stimulus intensity. Interestingly, there seems to exist a clear dissociation of the effects of both factors on RT and response force, disconfirming the view that both measures are merely two different sides of the same coin (see, e.g., Luce, 1986, p. 51). Response force increases with temporal uncertainty (compare Experiments 1 and 3) and stimulus intensity (Angel, 1973; Jaśkowski, Rybarczyk, Jaroszyk, \& Lemanski, 1995; Ulrich \& Mattes, 1996; Experiment 3 of the present study), whereas responses are slowed down by the former factor and speeded up by the latter factor. Although this dissociation is difficult to interpret within the framework of motor readiness, it nevertheless indicates that response force can be used as an inferential tool for uncovering basic principles of information processing.

\section{REFERENCES}

Abrams, R. A., \& Balota, D. A. (1991). Mental chronometry: Beyond reaction time. Psychological Science, 2, 153-157.

ANGEL, A. (1973). Input-output relations in simple reaction time experiments. Quarterly Journal of Experimental Psychology, 25, 193-200.

Bernstein, I. H., CHU, P. K., \& Briggs, P. (1973). Stimulus intensity and foreperiod effects in intersensory facilitation. Quarterly Journal of Experimental Psychology, 25, 171-181.

Bertelson, P., \& Tisseyre, F. (1969). The time-course of preparation: Confirmatory results with visual and auditory warning signals. Acta Psychologica, 30, 145-154.

Coles, M. G. H., Gratton, G., Bashore, T. R., Eriksen, C. W., \& Donchin, E. (1985). A psychophysiological investigation of the continuous flow model of human information processing. Journal of Experimental Psychology: Human Perception \& Performance, 11, 529-553.

GIRAY, M. (1990). Über die Aktivierung der menschlichen Motorik: Theoretische und experimentelle Analysen bei Reaktionsaufgaben [On the activation of the human motor system: Theoretical and experimental analysis of reaction time tasks]. Unpublished doctoral dissertation, Eberhard-Karls-Universität, Tübingen.

GIRAY, M., \& ULRICH, R. (1993). Motor coactivation revealed by response force in divided and focused attention. Journal of Experimental Psychology: Human Perception \& Performance, 19, 1278-1291.

HoHLE, R. H. (1965). Inferred components of reaction times as functions of foreperiod durations. Journal of Experimental Psychology, 69, 382-386.

JAŚKOWSKI, P., RYBARCZYK, K., JAROSZYK, F., \& LEMAŃSKI, D. (1995). The effect of stimulus intensity on force output in simple reaction time in humans. Acta Neurobiologiae Experimentalis, 55, 57-64.

JAŚKOWSKI, P., \& VERLEGER, R. (1993). A clock paradigm to study the relationship between expectancy and response force. Perceptual \& Motor Skills, 77, 163-174.

KaNTOWITZ, B. H. (1973). Response force as an indicant of conflict in double stimulation. Journal of Experimental Psychology, 100, 302-309.

Loftus, G. R., \& Masson, M. E. J. (1994). Using confidence intervals in within-subject designs. Psychonomic Bulletin \& Review, 1, 476-490.

LuCE, R. D. (1986). Response times: Their role in inferring elementary mental organization. New York: Oxford University Press.

MANNING, J. J., \& Hammond, G. R. (1990). The effect of motor preparation on changes in $\mathrm{H}$ reflex amplitude during the response latency of a warned reaction time task. Journal of Motor Behavior, 22, 292-314.

Meyer, D. E., Osman, A. M., Irwin, D. E., \& Yantis, S. (1988). Modern mental chronometry. Biological Psychology, 26, 3-67.

Miller, J. O., Franz, V., \& UlRich, R. (1997), Effects of auditory stim- ulus intensity on response force in simple, go-no-go, and choice RT. Manuscript submitted for publication.

MolenaAR, P. C. M., \& Van Der Molen, M. W. (1986). Steps to a formal analysis of the cognitive-energetic model of stress and human performance. Acta Psychologica, 62, 237-261

Mordkoff, J. T., Miller, J. O., \& Roch, A. C. (1996). Absence of coactivation in the motor component: Evidence from psychophysiological measures of target detection. Journal of Experimental Psychology: Human Perception \& Performance, 22, 24-41.

NÄ̈̈ÄNEN, R. (1971). Non-aging fore-periods and simple reaction time. Acta Psychologica, 35, 316-327.

NiEmI, P. (1979). Stimulus intensity effects on auditory and visual reaction processes. Acta Psychologica, 43, 299-312.

Niemi, P., \& Lethonen, E. (1982). Foreperiod and visual stimulus intensity: A reappraisal. Acta Psychologica, 50, 73-82.

Niemi, P., \& NÄÄTÄNEN, R. (1981). Foreperiod and simple reaction time. Psychological Bulletin, 89, 133-162.

NissEN, M. J. (1977). Stimulus intensity and information processing. Perception \& Psychophysics, 22, 338-352.

Posner, M. I., Nissen, M. J., \& KLEIN, R. M. (1976). Visual dominance: An information-processing account of its origins and significance. Psychological Review, 83, 157-171.

Requin, J., BREneR, J., \& RinG, C. (1991). Preparation of action. In J. R. Jennings \& M. G. H. Coles (Eds.), Handbook of cognitive psychophysiology: Central and autonomic nervous system approaches (pp. 357-448). New York: Wiley.

SANDERS, A. F. (1975). The foreperiod effect revisited. Quarterly Journal of Experimental Psychology, 27, 591-598.

SANDERS, A. F. (1977). Structural and functional aspects of the reaction process. In S. Dornic (Ed.), Attention and performance VI (pp. 3-25). Hillsdale, NJ: Erlbaum.

SANDERS, A. F. (1983). Towards a model of stress and human performance. Acta Psychologica, 53, 61-97.

SANDERS, A. F. (1990). Issues and trends in the debate on discrete vs. continuous processing of information. Acta Psychologica, 53, 61-97.

SANDERS, A. F., \& WerTHEIM, A. H. (1973). The relation between physical stimulus properties and the effect of foreperiod duration on reaction time. Quarterly Journal of Experimental Psychology, 25, 201206.

Stevens, S. S. (1955, October). Decibels of light and sound. Physics Today, $8,12-17$.

ULRICH, R., \& MATTES, S. (1996). Does immediate arousal enhance response force in simple reaction time? Quarterly Journal of Experimental Psychology, 49A, 972-990.

UlRICH, R., \& MilLER, J. O. (1994). Effects of truncation on reaction time analysis. Journal of Experimental Psychology: General, 123, 34-80.

UlRich, R., \& STAPF, K. H. (1984). A double-response paradigm to study stimulus intensity effects upon the motor system in simple reaction time experiments. Perception \& Psychophysics, 36, 545-558.

UlRICH, R., \& WING, A. M. (1991). A recruitment theory of force-time relations in the production of brief force pulses: The parallel force unit model. Psychological Review, 98, 268-294.

WINER, B. J. (1971). Statistical principles in experimental design. New York: McGraw-Hill.

Woodrow, H. (1914). The measurement of attention. Psychological Monographs, 17(5, Whole No. 76).

\section{NOTES}

1. To simplify matters, we have abbreviated the expression response stimulus to the word stimulus. Thus, stimulus never means "warning signal."

2. Hereafter, we use the term response preparation, since the meaning of subjective expectancy could be misleading in the present context.

3. The $S E M$ was estimated from the error terms $M S_{\mathrm{e}}$ of the repeated measures design (Loftus \& Masson, 1994); $S E=\sqrt{M S_{\mathrm{e}} / n}$, where $n$ is the number of scores averaged to get the corresponding mean at the level of the ANOVA. $M S_{\mathrm{e}}$ represents the pooled error terms for the factors foreperiod length, foreperiod regularity, and their interaction. 
4. According to Stevens (1955), the visual intensity levels of 0.3 and $100 \mathrm{~cd} / \mathrm{m}^{2}$ should correspond psychophysically to auditory intensity levels of 60 and $85 \mathrm{~dB}$, respectively. Thus, the weak visual stimulus was slightly stronger than the weak auditory stimulus. However, a pilot study suggested this slight increase to yield approximately equal mean RTs under both conditions.

5. In order to facilitate comparison with the preceding two experiments, stimulus modality had to be a between-subjects factor in this ex- periment. Thus, it is possible that this nonsignificant main effect reflects a loss in statistical power. However, this main effect is not of theoretical importance for addressing the questions that prompted Experiment 3.

(Manuscript received May 4, 1995;

revision accepted for publication October $9,1996$. 